\title{
水相中由壳聚糖-铜催化合成苯酚的方法研究
}

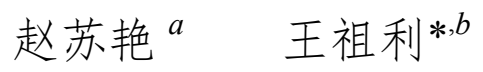 \\ ( ${ }^{a}$ 吉林警察学院实验技术中心 长春 130117) \\ $(b$ 青岛农业大学化学与药学院 青岛 266109)
}

\begin{abstract}
摘要 壳聚糖负载的铜催化剂催化下, 以苯嗍酸为原料, 在碱的水溶液中反应合成苯酚. 此方法反应条件温和, 操作 简单, 生成的苯酚产率高, 反应以水为溶剂, 对环境友好. 催化剂容易回收, 可以重复使用 5 次且催化剂的活性没有明 显降低.

关键词＼cjkstart壳聚糖负载铜催化剂; 可回收利用; 苯硼酸; 水溶剂
\end{abstract}

\section{Synthesis of Phenols under Mild Conditions in Water Using Recyclable Chitosan@Copper as Catalyst}

\author{
Zhao, Suyan ${ }^{a} \quad$ Wang, Zuli* ${ }^{*}, b$ \\ ( ${ }^{a}$ Jilin Police College Experimental Technology Center, Changchun 130117) \\ ( ${ }^{b}$ College of Chemistry and Pharmaceutical Sciences, Qingdao Agricultural University, Qingdao 266109)
}

\begin{abstract}
A green and efficient protocol for the synthesis of phenols using recyclable chitosan@copper as catalyst was developed. Phenols can be obtained in moderate to excellent yields. The catalyst can be recycled and reused for five times without significant loss of its catalytic activity.

Keywords chitosan@copper; recyclable; phenylboronic acid; water
\end{abstract}

苯酚及其衍生物由于其生物及药学活性, 是一类非 常重要的有机合成中间体 ${ }^{[1]}$, 广泛应用于农业、医药等 领域, 其合成研究也是合成领域里的一大热点. 工业上 合成苯酚的传统工艺方法是异丙苯法 ${ }^{[2]}$. 针对苯酚传统 工艺能耗大、工艺复杂、原子利用率低、苯酚收率不高、 环境污染严重等缺点, 苯酚的实验室合成方法是一般由 卤代芳烃亲核取代, 及铜催化剂催化重芳烃制备. 近年 来, 卤代芳烃羟基化合成苯酚成为了研究热点, 其中影 响比较广的途径的是由钯催化剂 ${ }^{[3]}$ 和铜催化剂 ${ }^{[4]}$ 催化的 卤代芳烃羟基化. 然而这些方法普遍存在对水和空气敏 感、金属试剂有毒、官能团相容性差、催化剂与产物不 能完全分离, 反应条件苛刻等缺点. 为了克服这些缺点, 化学家们一直致力于发展新的催化剂、反应试剂和新的 反应方法. 因此, 我们迫切需要尝试寻找一种新的催化 方法实现催化过程绿色化.

近年来, 非均相催化越来越受关注 ${ }^{[5]}$, 主要是非均
相催化剂易于与反应体系分离, 具有可回收、可以重复 利用等优点. 催化剂的载体材料有很多, 如碳材料、磁 性材料、二氧化硅及有机聚合物等 ${ }^{[6-8]}$. 随着人们对催 化剂研究的深入, 催化剂类型由金属负载转向了壳聚糖 负载, 壳聚糖固定化反应实现了很多碳碳键和碳硫键的 构成 ${ }^{[9 \sim 11]}$, 壳聚糖在有机合成领域正引起越来越多的关 注. 为继续倡导绿色化学反应, 寻找更为清洁高效的合 成苯酚的方法, 我们实现了以壳聚糖铜催化剂, 以水为 溶剂在温和的条件下对苯酚的合成.

\section{1 结果与讨论}

\section{1 反应条件的优化}

壳聚糖铜催化剂由文献方法合成 ${ }^{[10 b]}$, 首先我们以 苯硼酸做底物来考察反应(表 1). 在起初的篮选实验中, 我们尝试了碱、温度和反应时间对产率的影响, 选用碱

*E-mail: wangzulichem@163.com

Received October 23, 2015; revised November 16, 2015; published online December 10, 2015.

Project supported by the National Natural Science Foundation of China (No. 21402103), the Scientific Research Foundation of Shandong Province Outstanding Young Scientist Award (No. BS2013YY024), and the Postdoctoral Science Foundationof China (No. 150030).

国家自然科学基金(No. 21402103)、山东省优秀中青年基金(No. BS2013YY024)、中国博士后基金(No. 150030)资助项目. 
的分别有 $\mathrm{NaOH}, \mathrm{K}_{2} \mathrm{CO}_{3}, \mathrm{Na}_{2} \mathrm{CO}_{3}, \mathrm{~K}_{3} \mathrm{PO}_{4}, \mathrm{NaHCO}_{3}$ 和 $\mathrm{KOH}$ ，其中 $\mathrm{NaOH}$ 的反应产率最高(Entry 1), $\mathrm{KOH}$ 的反 应产率略低于 $\mathrm{NaOH}$ (Entry 4), 其它的碱的反应效果略 差(Entries 2, 3, 5, 6). 另外探讨了温度对反应的影响, 温 度从 40 到 $25{ }^{\circ} \mathrm{C}$ 反应产率依次明显降低(Entry 7), 比较 欣慰的是当温度升高到 $50{ }^{\circ} \mathrm{C}$ 时, 能得到一个比较高的 产率(Entry 8). 另外, 我们通过延长反应时间来探讨反 应时间对产率的影响. 结果发现当反应时间延长到 48 $\mathrm{h}$, 反应结果并无明显变化(Entry 9). 我们也对加入碱的 量对反应产率的影响做了研究. 加入碱的量加倍后反应 产率并没有明显提高.

\section{表 1 反应条件的优化 ${ }^{a}$}

Table 1 Optimization of the reaction conditions

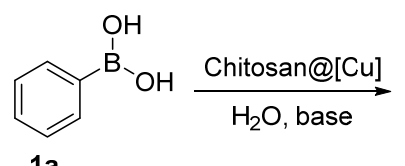<smiles>Oc1ccccc1</smiles>

2a

\begin{tabular}{llcl}
\hline Entry & Base & Temp. $/{ }^{\circ} \mathrm{C}$ & Yield/\% \\
\hline 1 & $\mathrm{NaOH}$ & 40 & 85 \\
2 & $\mathrm{~K}_{2} \mathrm{CO}_{3}$ & 40 & Trace \\
3 & $\mathrm{Na}_{2} \mathrm{CO}_{3}$ & 40 & Trace \\
4 & $\mathrm{KOH}_{5}$ & 40 & 78 \\
5 & $\mathrm{~K}_{3} \mathrm{PO}_{4}$ & 40 & Trace \\
6 & $\mathrm{NaHCO}_{3}$ & 40 & Trace \\
7 & $\mathrm{NaOH}$ & 25 & 36 \\
8 & $\mathrm{NaOH}$ & 50 & 93 \\
9 & $\mathrm{NaOH}$ & 50 & $94^{\mathrm{c}}$ \\
10 & $\mathrm{NaOH}$ & 50 & $92^{\mathrm{d}}$ \\
\hline
\end{tabular}

${ }^{a}$ 反应条件: 苯嗍酸(1a) $(1 \mathrm{mmol}) 、$ 碱 $(2 \mathrm{mmol}) 、$ 壳聚糖铜催化剂 $(125 \mathrm{mg})$ 、 水 $(1 \mathrm{~mL})$ 在空气中傥拌反应 $1 \mathrm{~h} .{ }^{b}$ 分离产率; ${ }^{c}$ 反应 $48 \mathrm{~h} ;{ }^{d}$ 氢氧化钠的量 3 mmol.

\section{2 底物范围的扩展}

在优化条件下, 本文拓展了一系列芳基嗍酸底物 (表 2), 芳基喼酸苯环上无论连有吸电子基团( $\mathbf{e} \sim \mathbf{2 n})$ 还 是供电子基团(2d, 2o, 2p, 2r), 都适用于该反应体系, 且 反应产率都很高. 然而由于基团的位阻作用, 2,6 二甲基 苯硼酸作底物的反应产率略低. 另外, 羰基、酯基、乙 酰基、氰基对本反应的耐受性都很好，因此反应物若含 有这些基团不容易受到破坏，由此可以为后续反应实现 官能团的构造. 除了苯环上含有取代基团的苯嗍酸外, 2-萘硼酸反应效果也很好 $(\mathbf{2 b}, \mathbf{2 c})$.

\section{3 催化剂的回收}

最后, 我们以苯嗍酸做底物探讨了催化剂的回收情 况. 反应结束后, 催化剂经过滤分离, 而后分别用乙醚 和丙酮淋洗. 最后在 $50{ }^{\circ} \mathrm{C}$ 下干燥 $4 \mathrm{~h}$, 可以直接下次使 用无需再纯化. 催化剂可以重复使用五次, 催化活性不
表 2 反应范围 ${ }^{a}$

Table 2 The scope of reaction

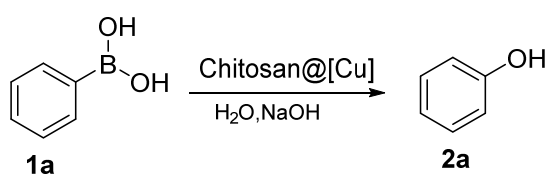

Entry 1

2

Yield $^{b} / \%$

2<smiles>Oc1ccc2ccccc2c1</smiles>

$1 \mathrm{~b}$

3<smiles>COc1cccc2ccccc12</smiles>

4<smiles>COc1ccc(C)cc1</smiles>

$1 d$

5<smiles>Oc1ccc(F)cc1</smiles>

6<smiles>Oc1ccc(Cl)cc1</smiles>

7<smiles>Oc1ccc(Br)cc1</smiles>

8<smiles>O=C(O)c1ccc(Br)cc1</smiles><smiles>O=C(O)c1ccc(O)cc1</smiles>

9<smiles>CC(=O)c1ccc(Br)cc1</smiles><smiles>Oc1ccc(Br)cc1</smiles>

93 86<smiles>CC(=O)c1ccc(O)cc1</smiles> 


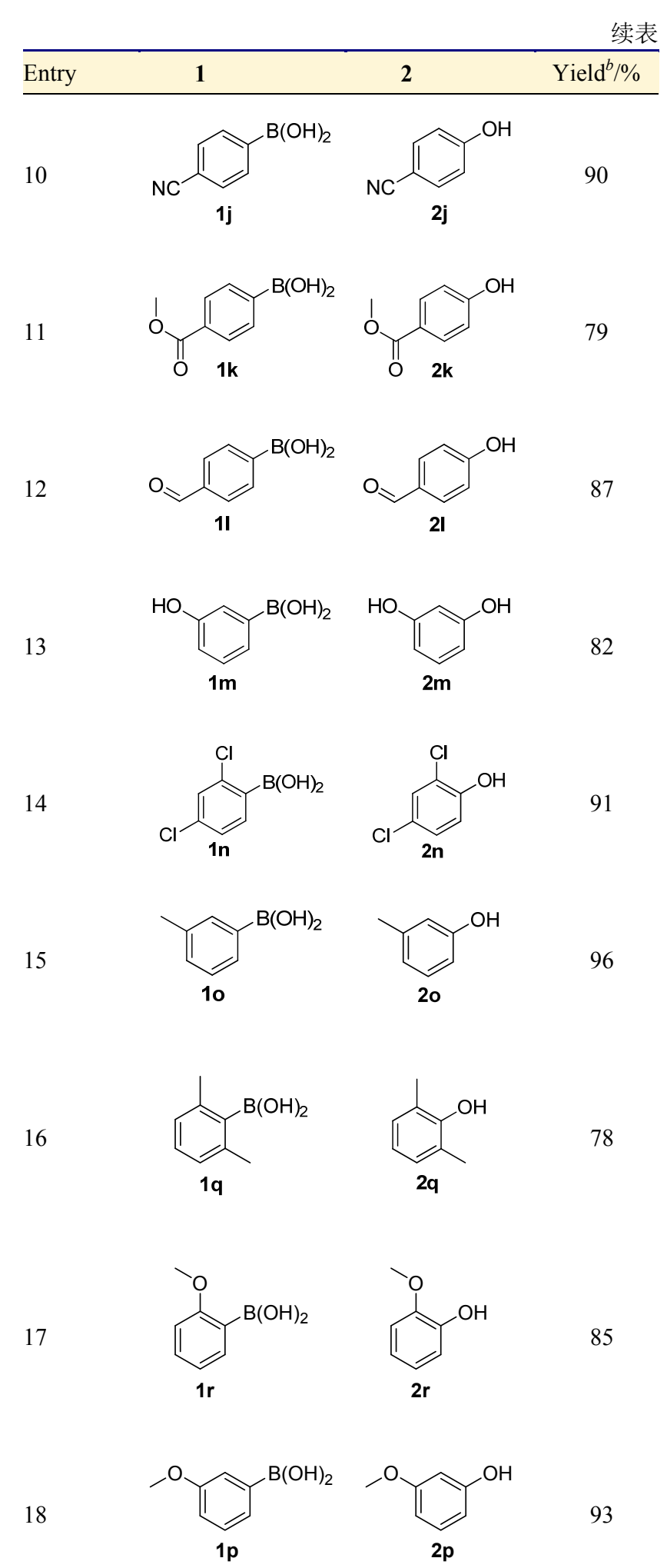

$\bar{a}$ 除非特殊注明, 反应物量均为 $1(1 \mathrm{mmol}), \mathrm{NaOH}(2 \mathrm{mmol})$ 和壳聚糖铜催 化剂 $(125 \mathrm{mg})$ 在水溶液中 $(1 \mathrm{~mL}) 50{ }^{\circ} \mathrm{C}$ 搅拌反应 $24 \mathrm{~h} ;{ }^{b}$ 分离产率.

会有明显降低. 等离子体发射光谱 (ICP)分析显示剩余 溶液里无铜离子的残留. ICP 分析反应溶液铜的浓度反 应前为 $5.80 \%$, 反应后浓度为 $5.79 \%$. 此结果证明壳聚 糖-铜催化剂稳定性比较强, 不容易受到破坏。
表 3 壳聚糖铜催化剂的回收再利用

Table 3 Recovery and reuse of chitosan@copper catalyst

\begin{tabular}{cc}
\hline 序号 & 产率/\% \\
\hline 1 & 93 \\
2 & 92 \\
3 & 94 \\
4 & 90 \\
5 & 91 \\
\hline
\end{tabular}

\section{2 结论}

本文研究了一种绿色以壳聚糖-铜为催化剂合成 苯酚的方法. 本方法不仅适用于苯硼酸, 而且还适用 于含有吸电子和供电子基团的取代芳基硼酸，反应 对氰基、酯基、羧基等官能团的耐受性都很好. 催化 剂稳定，经过滤后可以回收和重复使用。该方法具有 操作简单、底物适用范围广而且产物收率高等特点, 且溶剂为水，符合 “绿色化学” 的基本要求，为该类 化合物的广泛应用奠定了基础.

\section{3 实验部分}

\section{1 仪器与试剂}

核磁共振氢谱 ${ }^{1} \mathrm{H}$ NMR $(500 \mathrm{MHz})$ 和碳谱 ${ }^{13} \mathrm{C}$ NMR $(100 \mathrm{MHz})$ 采用 Bruker AV 500 核磁共振仪测定, 溶剂为 $\mathrm{CDCl}_{3}$ (内标为四甲基硅烷)，柱层析使用 $200 \sim 300$ 目硅 胶, 展开剂为石油醚和乙酸乙酯; 实验所用试剂均为市 售分析纯试剂, 均未进一步纯化处理.

\section{2 实验方法}

芳基硼酸 $(1 \mathrm{mmol}) 、$ 碱 $(2 \mathrm{mmol}) 、$ 壳聚糖铜催化剂 $(125 \mathrm{mg})$ 、水 $(1 \mathrm{~mL})$ 置于 $25 \mathrm{~mL}$ 的真空管中, 在空气中 加热 $50{ }^{\circ} \mathrm{C}$ 搅拌 $24 \mathrm{~h}$, TLC 检测反应. 反应结束后, 反 应溶液用 $10 \mathrm{~mL}$ 乙醚萃取 3 次, 合并有机相, 并用无水 硫酸钠干燥, 然后减压旋蒸去除溶剂, 剩余物用硅胶柱 色谱分离纯化, 用石油醚和乙酸乙酯作洗脱剂, 得目标 产物。

苯酚(2a) ${ }^{[12]}:{ }^{1} \mathrm{H}$ NMR $\left(500 \mathrm{MHz}, \mathrm{CDCl}_{3}\right) \delta: 7.29(\mathrm{t}$, $J=7.2 \mathrm{~Hz}, 2 \mathrm{H}), 6.98(\mathrm{t}, J=6.8 \mathrm{~Hz}, 1 \mathrm{H}), 6.88(\mathrm{~d}, J=7.5$ $\mathrm{Hz}, 2 \mathrm{H}), 5.24$ (br s, $1 \mathrm{H}) ;{ }^{13} \mathrm{C} \mathrm{NMR}\left(\mathrm{CDCl}_{3}, 500 \mathrm{MHz}\right) \delta$ : 155.3, 129.7, 120.9, 115.3.

2-菜酚 $(\mathbf{2 b})^{[13]}:{ }^{1} \mathrm{H}$ NMR $\left(500 \mathrm{MHz}, \mathrm{CDCl}_{3}\right) \delta: 7.80 \sim$ $7.77(\mathrm{~m}, 2 \mathrm{H}), 7.78 \sim 7.70(\mathrm{~m}, 1 \mathrm{H}), 7.47 \sim 7.44(\mathrm{~m}, 1 \mathrm{H})$, $7.37 \sim 7.34(\mathrm{~m}, 1 \mathrm{H}), 7.17(\mathrm{~s}, 1 \mathrm{H}), 7.13 \sim 7.12(\mathrm{~m}, 1 \mathrm{H})$, $4.96(\mathrm{~s}, 1 \mathrm{H}) ;{ }^{13} \mathrm{C} \mathrm{NMR}\left(\mathrm{CDCl}_{3}, 500 \mathrm{MHz}\right) \delta: 153.6,134.5$, $129.8,128.9,127.7,126.5,126.3,123.6,117.7,109.4$.

1-蒜酚 $(2 \mathrm{c})^{[14]}:{ }^{1} \mathrm{H}$ NMR $\left(500 \mathrm{MHz}, \mathrm{CDCl}_{3}\right) \delta: 7.85$ (m, 2H), $7.49 \sim 7.47(\mathrm{~m}, 1 \mathrm{H}), 7.36 \sim 7.29(\mathrm{~m}, 1 \mathrm{H}), 6.84 \sim$ 
$6.85(\mathrm{dd}, J=7.4 \mathrm{~Hz}, 1 \mathrm{H}), 5.29(\mathrm{~s}, 1 \mathrm{H}) ;{ }^{13} \mathrm{C} \mathrm{NMR}\left(\mathrm{CDCl}_{3}\right.$, $500 \mathrm{MHz}) \delta$ : 151.4, 134.7, 127.7, 126.5, 125.8, 125.3, $121.5,120.7,108.6$.

对甲基苯酚(2d) ${ }^{[15]}$ : ${ }^{1} \mathrm{H}$ NMR (500 $\left.\mathrm{MHz}, \mathrm{CDCl}_{3}\right) \delta$ : 7.07 (d, $J=8.2 \mathrm{~Hz}, 2 \mathrm{H}), 6.76$ (d, $J=8.4 \mathrm{~Hz}, 2 \mathrm{H}), 4.65$ (br $\mathrm{s}, 1 \mathrm{H}), 2.30(\mathrm{~s}, 3 \mathrm{H}) ;{ }^{13} \mathrm{C} \mathrm{NMR}\left(\mathrm{CDCl}_{3}, 500 \mathrm{MHz}\right) \delta: 153.2$, 130.0, 130.0, 115.0, 20.5.

4-氟苯酚 $(2 \mathrm{e})^{[16]}:{ }^{1} \mathrm{H}$ NMR $\left(500 \mathrm{MHz}, \mathrm{CDCl}_{3}\right) \delta$ : $6.94 \sim 6.90(\mathrm{~m}, 2 \mathrm{H}), 6.78 \sim 6.75(\mathrm{~m}, 2 \mathrm{H}), 4.74(\mathrm{bs}, 1 \mathrm{H})$; ${ }^{13} \mathrm{C} \mathrm{NMR}\left(\mathrm{CDCl}_{3}, 500 \mathrm{MHz}\right) \delta: 158.2,156.3,151.5,116.2$, 116.1, 115.8 .

4-氯苯酚 $(2 \mathbf{f})^{[15]}:{ }^{1} \mathrm{H}$ NMR $\left(500 \mathrm{MHz}, \mathrm{CDCl}_{3}\right) \delta: 7.20$ (d, $J=9.0 \mathrm{~Hz}, 2 \mathrm{H}), 6.80$ (d, $J=8.9 \mathrm{~Hz}, 2 \mathrm{H}$ ), 5.56 (br s, $1 \mathrm{H}) ;{ }^{13} \mathrm{C} \mathrm{NMR}\left(\mathrm{CDCl}_{3}, 500 \mathrm{MHz}\right) \delta: 153.8,129.7,125.8$, 116.8 .

4-溴苯酚 $(\mathbf{2 g})^{[17]}:{ }^{1} \mathrm{H}$ NMR $\left(500 \mathrm{MHz}, \mathrm{CDCl}_{3}\right) \delta: 7.20$ (d, $J=9.0 \mathrm{~Hz}, 2 \mathrm{H}), 6.64$ (d, $J=8.9 \mathrm{~Hz}, 2 \mathrm{H}), 5.22$ (br s, $1 \mathrm{H}) ;{ }^{13} \mathrm{C} \mathrm{NMR}\left(\mathrm{CDCl}_{3}, 500 \mathrm{MHz}\right) \delta: 153.6,131.5,116.2$, 111.8 .

4-羟基苯甲酸(2h) ${ }^{[15]}:{ }^{1} \mathrm{H} \mathrm{NMR}\left(500 \mathrm{MHz}, \mathrm{CDCl}_{3}\right) \delta$ : $8.13(\mathrm{~d}, J=7.1 \mathrm{~Hz}, 2 \mathrm{H}), 7.63 \sim 7.60(\mathrm{~m}, 1 \mathrm{H}), 7.50 \sim 7.46$ $(\mathrm{m}, 2 \mathrm{H}) ;{ }^{13} \mathrm{C} \mathrm{NMR}\left(\mathrm{CDCl}_{3}, 500 \mathrm{MHz}\right) \delta: 172.3,133.8$, 130.2, 129.3,128.5.

4-羟基苯乙酮 $(\mathbf{2 i})^{[15]}:{ }^{1} \mathrm{H} \mathrm{NMR}\left(\left(500 \mathrm{MHz}, \mathrm{CDCl}_{3}\right) \delta\right.$ : $7.93(\mathrm{~d}, J=6.5 \mathrm{~Hz}, 2 \mathrm{H}), 6.93$ (d, $J=8.5 \mathrm{~Hz}, 2 \mathrm{H}), 6.59$ (s, $1 \mathrm{H}), 6.59(\mathrm{~s}, 1 \mathrm{H}), 2.59(\mathrm{~s}, 3 \mathrm{H}) ;{ }^{13} \mathrm{C} \mathrm{NMR}\left(\mathrm{CDCl}_{3}, 500\right.$ MHz) $\delta: 207.7,197.4,160.5,131.0,115.3,31.0$.

4-羟基苯乙腈 $(\mathbf{2} \mathbf{j}){ }^{[15]}:{ }^{1} \mathrm{H} \mathrm{NMR}\left(500 \mathrm{MHz}, \mathrm{CDCl}_{3}\right) \delta$ : 7.58 (d, $J=8.2 \mathrm{~Hz}, 2 \mathrm{H}), 7.28$ (s, 1H), 6.93 (d, $J=8.7 \mathrm{~Hz}$, $2 \mathrm{H}) ;{ }^{13} \mathrm{C} \mathrm{NMR}\left(\mathrm{CDCl}_{3}, 500 \mathrm{MHz}\right) \delta: 159.9,134.3,116.4$, 103.5 .

4-羟基苯甲酸甲酯 $(\mathbf{2 k})^{[15]}$ : ${ }^{1} \mathrm{H}$ NMR $(500 \mathrm{MHz}$, $\left.\mathrm{CDCl}_{3}\right) \delta: 7.96(\mathrm{~d}, J=8.8 \mathrm{~Hz}, 2 \mathrm{H}), 6.87(\mathrm{~d}, J=8.8 \mathrm{~Hz}$, 2H), 5.80 (br s, $1 \mathrm{H}), 3.89$ (s, 3H); ${ }^{13} \mathrm{C} \mathrm{NMR}\left(\mathrm{CDCl}_{3}, 500\right.$ $\mathrm{MHz}) \delta: 167.0,159.9,132.0,122.6,115.2,52.0$.

2,4-二氯苯酚(2n) ${ }^{[16]}:{ }^{1} \mathrm{H} \mathrm{NMR}\left(500 \mathrm{MHz}, \mathrm{CDCl}_{3}\right) \delta$ : $7.35(\mathrm{~d}, J=4.5 \mathrm{~Hz}, 2 \mathrm{H}), 7.18(\mathrm{~d}, J=8.8 \mathrm{~Hz}, 2 \mathrm{H}), 6.98(\mathrm{~m}$, $1 \mathrm{H}), 5.52(\mathrm{~s}, 1 \mathrm{H}) ;{ }^{13} \mathrm{C} \mathrm{NMR}\left(\mathrm{CDCl}_{3}, 500 \mathrm{MHz}\right) \delta: 150.2$, 128.6, 128.5, 125.6, 120.4, 117.1.

间苯二酚 $(\mathbf{2 m})^{[16]}:{ }^{1} \mathrm{H}$ NMR $\left(500 \mathrm{MHz}, \mathrm{CDCl}_{3}\right) \delta$ : 7.24 (t, $J=7.0 \mathrm{~Hz}, 3 \mathrm{H}), 6.93$ (t, $J=7.4 \mathrm{~Hz}, 1 \mathrm{H}), 6.84$ (d, $J=8.5 \mathrm{~Hz}, 2 \mathrm{H}), 5.12(\mathrm{~s}, 1 \mathrm{H}) ;{ }^{13} \mathrm{C} \mathrm{NMR}\left(\mathrm{CDCl}_{3}, 500 \mathrm{MHz}\right)$ $\delta: 155.4,129.7,120.8,115.3$.

4-羟基苯甲醛(2l) ${ }^{[15]}:{ }^{1} \mathrm{H} \mathrm{NMR}\left(500 \mathrm{MHz}, \mathrm{CDCl}_{3}\right) \delta$ : 9.88 (s, 1H), 7.82 (d, $J=8.7 \mathrm{~Hz}, 2 \mathrm{H}), 6.98$ (d, $J=8.6 \mathrm{~Hz}$,
2H), $6.20(\mathrm{~s}, 1 \mathrm{H}) ;{ }^{13} \mathrm{C} \mathrm{NMR}\left(\mathrm{CDCl}_{3}, 500 \mathrm{MHz}\right) \delta: 191.1$, $161.5,132.5,129.9,116.0$.

3-甲基苯酚(2o) ${ }^{[14]}:{ }^{1} \mathrm{H} \mathrm{NMR}\left(500 \mathrm{MHz}, \mathrm{CDCl}_{3}\right) \delta$ : $7.11(\mathrm{t}, J=7.8 \mathrm{~Hz}, 1 \mathrm{H}), 6.75(\mathrm{~d}, J=7.5 \mathrm{~Hz}, 1 \mathrm{H}), 6.65 \sim$ $6.62(\mathrm{~m}, 1 \mathrm{H}), 4.75(\mathrm{~s}, 1 \mathrm{H}), 2.30(\mathrm{~s}, 3 \mathrm{H}) ;{ }^{13} \mathrm{C} \mathrm{NMR}\left(\mathrm{CDCl}_{3}\right.$, $500 \mathrm{MHz}) \delta: 155.4,139.8,129.4,121.6,116.0,112.2,21.3$.

3-甲氧基苯酚(2p) ${ }^{[14]}:{ }^{1} \mathrm{H} \mathrm{NMR}\left(500 \mathrm{MHz}, \mathrm{CDCl}_{3}\right) \delta$ : $7.16(\mathrm{~m}, 1 \mathrm{H}), 6.53(\mathrm{dd}, J=8.1 \mathrm{~Hz}, 1 \mathrm{H}), 6.47 \sim 6.44(\mathrm{~m}$, 2H), $5.29(\mathrm{~s}, 1 \mathrm{H}), 3.81(\mathrm{~s}, 3 \mathrm{H}) ;{ }^{13} \mathrm{C} \mathrm{NMR}\left(\mathrm{CDCl}_{3}, 500\right.$ $\mathrm{MHz}) \delta: 160.9,156.7,130.2,107.8,106.5,101.6,55.3$.

2,6-二甲基苯酚(2q) ${ }^{[14]}$ : ${ }^{1} \mathrm{H}$ NMR $\left(500 \mathrm{MHz}, \mathrm{CDCl}_{3}\right)$ $\delta: 7.07$ (d, $J=7.5 \mathrm{~Hz}, 2 \mathrm{H}), 6.86(\mathrm{t}, J=7.5 \mathrm{~Hz}, 1 \mathrm{H}), 4.63$ (br s, $1 \mathrm{H}), 2.28(\mathrm{~s}, 6 \mathrm{H}) ;{ }^{13} \mathrm{C} \mathrm{NMR}\left(\mathrm{CDCl}_{3}, 500 \mathrm{MHz}\right) \delta$ : 152.2, 128.6, 123.0, 120.2,15.8.

邻甲氧基苯酚 $(2 r){ }^{[14]}:{ }^{1} \mathrm{H}$ NMR $\left(500 \mathrm{MHz}, \mathrm{CDCl}_{3}\right) \delta$ : $7.25(\mathrm{~d}, J=7.8 \mathrm{~Hz}, 1 \mathrm{H}), 7.09 \sim 7.02(\mathrm{~m}, 2 \mathrm{H}), 6.96 \sim 6.94$ $(\mathrm{m}, 1 \mathrm{H}), 6.73(\mathrm{~s}, 1 \mathrm{H}), 3.82(\mathrm{~s}, 3 \mathrm{H}) ;{ }^{13} \mathrm{C} \mathrm{NMR}\left(\mathrm{CDCl}_{3}, 500\right.$ $\mathrm{MHz}) \delta: 147.2$, 146.0, 121.6, 120.6, 115.2, 111.4, 55.8.

辅助材料(Supporting Information) 合成产物的 ${ }^{1} \mathrm{H}$ $\mathrm{NMR}$ 和 ${ }^{13} \mathrm{C} \mathrm{NMR}$ 谱图, 这些材料可以免费从本刊网 站(http://sioc-journal.cn/)上下载.

\section{References}

[1] Rappoport, Z. The Chemistry of Phenols, Wiley-VCH, Weinheim, Germany, 2003.

[2] Zhang, R. X.; Zhang, X. D. Chemical Industry 2008, 26, 47 (in Chinese). (张日新, 张晓东, 化学工业, 2008, 26, 47.)

[3] (a) Anderson, K. W.; Ikawa, T. R.; Tundel, E. S.; Buchwald, L. J. Am. Chem. Soc. 2006, 128, 10694.

(b) Willis, M. C. Angew. Chem., Int. Ed. 2007, 46, 3402

(c) Sergeev, A. G.; Schulz, T.; Torborg, C.; Spannenberg, A.; Neumann, H.; Beller, M. Angew. Chem., Int. Ed. 2009, 48, 7595.

(d) Schulz, T.; Torborg, C.; Schäffner, B.; Huang, J.; Zapf, A.; Kadyrov, R.; Börner, A.; Beller, M. Angew. Chem., Int. Ed. 2009, 48, 918 .

(e) Gallon, B. J.; Kojima, R. W.; Kaner, R. B.; Diaconescu, P. L. Angew. Chem., Int. Ed. 2007, 46, 7251.

(f) Chen, G. S.; Chan, A. S. C.; Kwong, F. Y. Tetrahedron Lett. 2007, 48, 473.

[4] (a) Yang, D. S.; Fu, H. Chem. Eur. J. 2010, 16, 2366.

(b) Kormos, C. M.; Leadbeater, N. E. Tetrahedron 2006, 62, 4728; (c) Tlili, A.; Xia, N.; Monnier, F.; Taillefer, M. Angew. Chem., Int. Ed. 2009, 48, 8725 .

(d) Zhao, D. B.; Wu, N. J.; Zhang, S.; Xi, P.; Su, X. Y.; Lan, J. B.; You, J. S. Angew. Chem., Int. Ed. 2009, 48, 8729.

[5] Yin, L.; Leibescher, J. Chem. Rev. 2007, 107, 133.

[6] Li, B.; Li, M.; Yao, C. H.; Shi, Y. F.; Ye, D. R.; Wu, J.; Zhao, D. Y. J. Mater. Chem. 2013, 1, 6742.

[7] For selected examples, see: (a) Kantam, M. L.; Yadav, Y.; Laha, Y.; Srinivas, P.; Sreedhar, B.; Figueras, F. J. Org. Chem. 2009, 74, 4608 . 
(b) Kundu, D.; Chatterjee, T.; Ranu, B. C. Adv. Synth. Catal. 2013, $355,2285$.

(c) Brahmachari, G.; Laskar, S.; Barik, P. RSC Adv. 2013, 3, 142;

(d) Parella, R.; Kumar, A; Babu, S. A. Tetrahedron Lett. 2013, 54, 1738.

(e) Yang, S.; Wu, C.; Zhou, H.; Yang, Y.; Zhao, Y.; Wang, C.; Yang, W.; Xu, J. Adv. Synth. Catal. 2013, 355, 53.

(f) Swapna, K.; Murthy, S. N.; Jyothi, M. T.; Nageswar, Y. V. D. Org. Biomol. Chem. 2011, 5989.

(g) Hudson, R.; Ishikawa, S.; Li, C.-J.; Moores, A. Synlett 2013, 1637.

(h) Dandia, A.; Jain, A. K.; Sharma, S. RSC Adv. 2013, 3, 2924.

(i) Kumar, A. S.; Reddy, M.; M.Knorn, A.; Reiser, O.; Sreedhar, B. Eur. J. Org. Chem. 2013, 4, 674.

(j) Wang, Z. L. RSC Adv. 2015, 5, 5563.

[8] Zhang, J.; Han, D.; Zhang, H.; Chaker, M.; Zhao, Y.; Ma, D. Chem. Commun. 2012, 48, 11510.

[9] (a) Makhubela, B. C. E.; Jardine, A.; Smith, G. S. Appl. Catal. 2011, 393, 231.

(b) Lasri, J.; Leod, T. C. O. M.; Pombeiro, A. J. L. Appl. Catal.
2011, 393, 94

(c) Yi, S. S.; Lee, D. H.; Sin, E.; Lee, Y. S. Tetrahedron Lett. 2007, $48,6771$.

[10] (a) Shen, C.; Xu, J.; Yu, W.; Zhang, P. Green Chem. 2014, 16, 3007. (b) Shen, C.; Xu, J.; Yu, W. B.; Zhang, P. F. Green Chem. 2014, 16, 3007.

[11] Yang, B.; Mao, Z. X.; Zhu, X. H.; Wan, Y. Q. Catal. Commun. 2015, 60, 92.

[12] Pal, M.; Parasuraman, K.; Yeleswarapu, K. R. Org. Lett. 2003, 5, 349.

[13] Molander, G. A.; Cavalcanti, L. N. J. Org. Chem. 2011, 76, 623.

[14] Jiang, M.; Yang, H. J.; Li, Y.; Jia, Z. Y.; Fu, H. Chin. Chem. Lett. 2014, 25, 715 .

[15] Yang, D. S.; An, B. J.; Wei, W.; Jiang, M.; You, J. M.; Wang, H. Tetrahedron 2014, 70, 3630.

[16] Xu, J. M.; Wang, X. Y.; Shao, C. W.; Su, D. Y.; Cheng, G. L.; Hu, Y. F. Org. Lett. 2010, 12, 164.

[17] Tlili, A.; Xia, N.; Monnier, F.; Taillefer, M. Angew. Chem., Int. Ed. $\mathbf{2 0 0 9}, 48,8725$. 